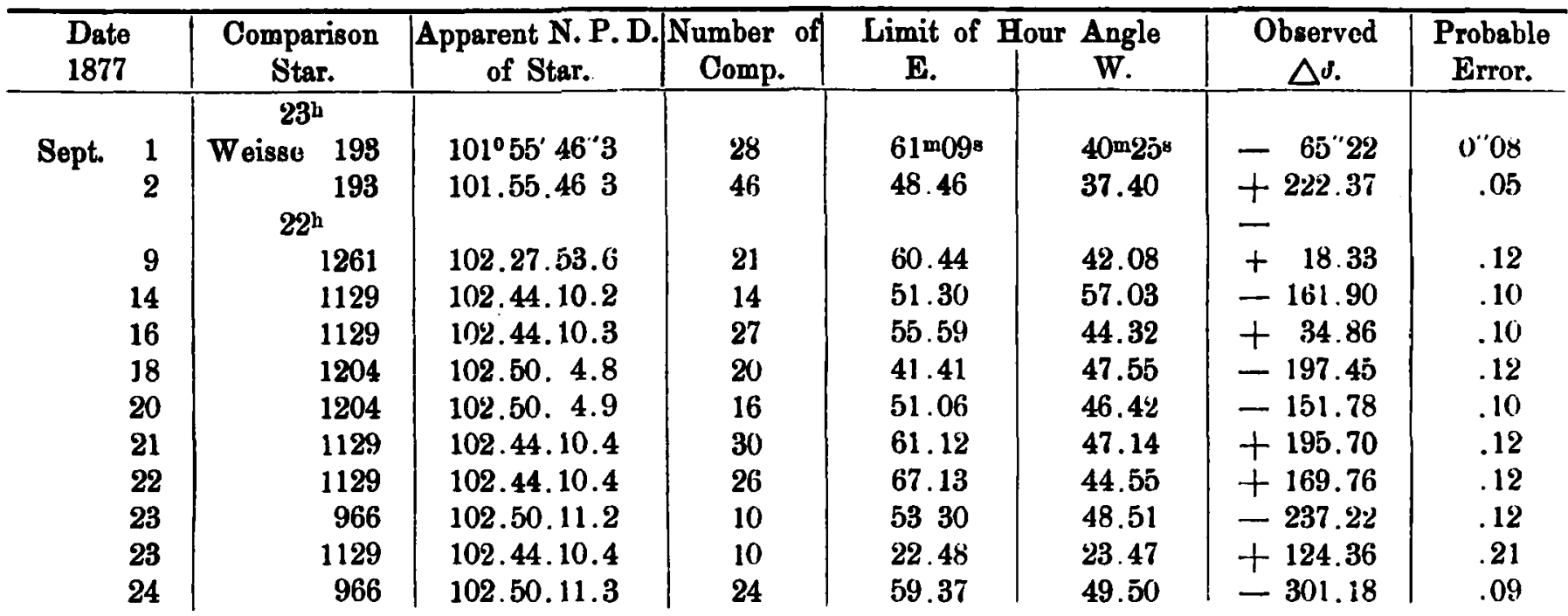

The above North Polar Distances are computed roughly from Weisse's Bessel.

Lehigh University, South Bethlehem, Pennsylvania. 1877 Dcc. 12.

C. L. Doolittle.

\title{
Beobachtungen des Planeten Mars sammt Vergleichssternen
}

zu Kremsmünster im Jahre 1877.

7. Aug. Weisse $76 \quad a=23^{\text {h }} 6^{\mathrm{m}} 37^{8} 63$ $\psi^{2}$ Aquarii Mars

B. A. C. 8239

B. A. C. 8266

B. A. C. 8285

13. Aug. 74 Aquarii

$h^{2}$ Aquarii

Weisse 76

$\psi^{2}$-Aquarii

Mars

B. A. C. 8239

B. A. C. 8266

B. A. C. 8285

Weisse 985

6. Sept. 64 Aquarii

70 Aquarii

74 Aquarii

Mars

Weisse 394

B. A. C. 8199

B. A. C. 8221

B. A. C. 8266
23.11.34.31 (Eph. - a)

23.28.34.78 d $\alpha=+0825$

23.34 .50 .76

23.40 .59 .47

23.43 .57 .48

22.47. 3.87

22.58.48.68

23. 6.37 .63

23.11.34.51

23.27 .21 .90

23.34.50.59

23.40 .59 .49

23.43 .57 .58

23.49.30.32

22.32 .51 .88

22.42. 6.00

22.47 .4 .20

23. 7.38 .80

23.21. 6.20

23.25 .54 .05

23.31.21.04

23.41. 0.06 $\delta=-10^{\circ} 13^{\prime} 58^{\prime \prime} 3$

9:50.52.2 (Eph. - $\delta$ ) m. Kr. Z.

9.59.31.0 d $\delta=+5^{\prime \prime} 6 \pi=17^{\prime \prime} 30,14^{\mathrm{h}} 21^{\mathrm{m}} 55^{\mathrm{s}} 46$

12.21 .22 .0

12.35. 4.1

10.39 .14 .4

12.15 .51 .5

8.21 .23 .0

10.13 .57 .4

9.50 .50 .8

10.19 .57 .3

12.21 .20 .3

12.35. 3.4

10.39 .13 .6

13.49.39.9

10.39 .42 .3

11.11 .57 .4

12.15 .50 .2

$12.15 .47 .0+5.6 \pi=19.70,12^{\mathrm{h}} 3^{\mathrm{m}} 5^{\mathrm{s}} 63$

13.35 .59 .4

12.12 .57 .5

13.44 .8 .0

12.35. 5.4
$+4.8 \pi=18.11,13^{\mathrm{h}} 57^{\mathrm{m}} 7^{\mathrm{s} 31}$ 
14. Sept. 64 Aquarii 70 Aquarii 74 Aquarii B. A. C. 8004 Mars Weisse 394 B. A. C. 8199 B. A. C. 8221 B. A. C. 8266

11. Sept. 64 Aquarii 70 Aquarii 74 Aquarii B. A. C. 8004 Mars

Weisse 394

B. A. C. 8199

B. A. C. 8221

B. A. C. 8266

23. Sept. 64 Aquarii

70) Aquarii 74 Aquarii Mars Weisse 394

B. A. C. 8199

B. A. C. 8221

B. A. C. 8266

26. Sept. $\sigma$ Aquarii 64 Aquarii 70 Aquarii Mars

Weisse 1241 Weisse 76 4 Aquarii

27. Sept. 50 Aquarii - Aquarii 64 Aquarii 70 Aquarii Mars Weisse 1241 Weisse 76 $\psi^{2}$ Aquarii

28. Sept. o Aquarii 64 Aquarii 70 Aquarii

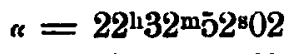

$$
\begin{aligned}
& \text { 22.42. } 6.05 \\
& \text { 22.47. } 4.32 \\
& \text { 22.03.11.25 (Eph. - }) \\
& \text { 22.59.14.55 } \mathrm{d} a=+0345 \\
& \text { 23.21. } 6.21 \\
& \text { 23.25.54.11) } \\
& 23.31 .21 .01 \\
& 2341.0 .10
\end{aligned}
$$

22.32 .51 .96

22.42 . 5.95

22.47. 4.16

2253.11 .18

23. 2.17.21

$23.21 \quad 6.26$

23.25 .54 .00

23.21 .20 .98

23.41 . 0.02

22.32 .52 .00

2242.6 .15

22.47. 4.41

22.51 .39 .96

23.21.05.99

23.25 .54 .20

23.31.21.20

23.41. 0.07

22.24 .12 .33

22.32.51.86

22.42. 6.00

22.49 .49 .85

22.59 .55 .50

23. 6.37 .94

23.11.34.65

22.17 .55 .98

22.24 .12 .33

22.32 .51 .87

22.42 .5 .94

22.49 .18 .60

22.59 .55 .55

23. 6.37 .93

23.11 .34 .70

22.24.12.29

22.32 .51 .88

22.42. 6.01

$$
\begin{aligned}
& \delta=-10^{\circ} 39^{\circ} 43^{\prime \prime} 0 \\
& 11.11 .57 .0 \\
& \text { 12.15.50.6 } \\
& \text { 13.43.27.4 (Eph. - J) m. Kr. Z. } \\
& 12.40 .44 .0 \mathrm{~d} \delta=+5^{\prime \prime} 6 \pi=19^{\prime \prime} 30,11^{\mathrm{h}} 23^{\mathrm{m}} 15^{\mathrm{s} 52} \\
& 13.35 .57 .0 \\
& 12.12 .58 .5 \\
& \text { 13.44. } 5.5 \\
& 1235.4 .4
\end{aligned}
$$

10.39 .41 .8

11.11 .56 .9

12.15. 49.9

13.43.26.7

12.33 .21 .4

13.35. 58.2

$+5.5 \pi=19.58,11^{\mathrm{b} 38^{\mathrm{m}}} 5^{\mathrm{8} 38}$

12.12 .58 .0

13.44. 6.5

12.35. 5.3

10.39.43.6

11.11 .56 .8

12.15 .51 .2

$+0.42$

12.46 .15 .0

13.35 .58 .6

$+6.9_{\pi}=18.32,10^{\mathrm{h}} 40^{\mathrm{m}} 18^{8} 98$

12.12 .59 .0

13.44. 7.0

14.35. 5.2

$11.18 \quad$ ว. 7

11). 39.42 .6

11.11 .56 .2

$+0.50 \quad 12.4219 .4$

10.14 .42 .8

$+8.0 \pi=17.86,10^{\mathrm{h}} 26^{\mathrm{m}} 41^{\mathrm{s}} 45$

10.13 .58 .5

$9 . \overline{0} 050 . \overline{0}$

14. $8 . \overline{0} 4.6$

11.18 .6 .5

10.39.43.4

$11.11 . \overline{0} 6.7$

$+0.41 \quad 12.40 .22 .6$

$+8 . \overline{\mathrm{g}} \pi=17.7 \overline{\mathrm{D}}, 10^{\mathrm{h} 22^{\mathrm{m}} 14^{\mathrm{s}} 37}$

10.14 .44 .0

10.13 .58 .0

9.5ก.ริ) 9

11.18. 6.4

10.39 .43 .7

11.11 .56 .3 
Mars

Weisse 1241

Weisse 76

$\psi^{2}$ Aquarii

97 Aquarii

29. Sept. 50 Aquarii

- Aquarii

64 Aquarii

70 Aquarii

Mars

Weisse 1241

Weisse 76

$y^{2}$ Aquarii

97 Aquarii

14. Oct. 50 Aquarii

- Aquarii

64 Aquarii

70 Aquarii

Mars

Weisse 1241

Weisse 76

$\psi^{2}$ Aquarii

97 Aquarii

15. Oct. $\sigma$ Aquarii

64 Aquarii

70 Aquarii

Mars

Weisse 1241

Weisse 76

$\psi^{2}$ Aquarii

97 Aquarii

19. Oct. Aquarii

64 Aquarii

70 Aquarii

Mars

Weisse 1241

Weisse 76

$\psi^{2}$ Apuarii

97 Aquarii

20. Oct. 50 Aquarii

a Aquarii

64 Aquarii

70) Aquarii
(Eph. -

(Eph. - $-\delta)$

m. Kr. Z.

$a=22^{4} 48^{\mathrm{m} 50} \mathrm{~s}\left(10 \mathrm{~d} \alpha=+0^{\prime \prime} 41 d=-12^{\circ} 38^{\prime} 6^{\prime \prime} 1 \mathrm{~d} d=+8^{\prime \prime} 3 \pi=17^{\prime \prime} 59,10^{\mathrm{h}} 17^{\mathrm{m}} 4.9895\right.$ 22.5955 .62

23. 6.38 .00

23. 11.34 .80

23.16.16.46

22.17 .55 .92

22.24 .12 .45

22.32 .51 .86

22.42 .598

22.48 .24 .20

22.59 .55 .58

23. 6.38 .05

23.11 .34 .78

23.16.16.4l

$22.17 .5 \overline{5} .92$

22.24 .12 .35

22.32 .51 .72

2242.5 .90

22.47 .54 .10

$22.59 .55 .50)$

23. 6.38 .02

23.11 .34 .73

23.16 .16 .48

$+0.45$

22.24 .12 .32

22.32 .51 .67

2242.583

22.48 .1585

22.59 .55 .40

23. 6.38 .02

23.11.34.65

23.16 .16 .48

22.24 .12 .22

22.32 .51 .60

22.42. 5.73

22.50 .10 .30

22.59 .55 .32

23. $6.37 .8 y$

23.11.34.64

23.16.16.45

22.17 .55 .82

22.24 .12 .23

22.32 .51 .66

22. 42.5 .74
$+0.35$

10.14 .45 .2

10.13 .58 .8

9.50 .49 .7

15.42 .28 .3

14. 8.54 .3

11.18 .7 .0

10.39 .44 .4

11.11 .56 .9

12.3530 .6

10.14 .45 .3

10.1357 .6

9.50 .50 .5

15.42 .28 .4

14. 8.549

11.18. 9.3

11). 39.45 .2

11.11 .57 .9

11.20 .55 .8
$+0.38 \quad 11.256 .3$

10.1446 .3

10.13 .59 .0

9 50.52 .0

15.42 .30 .0

11.18. 9.1

10.39 .46 .4

11.11 .58 .8

11.13 .46 .6

10. 14.47 .3

10.14.0.6

9.5053 .5

1542.30 .0

11.18. 8.4

10.39 .46 .0

11.11 .57 .6

$+0.41$

10.42 .47 .7

10.14 .46 .3

10.14.0.9

9.50.54.4

15. 42.29 .4

14. 8.53 .0

11.18 .8 .1

10.39 .45 .6

11.11 .58 .1
$+7.9 \pi=17.44,20^{\mathrm{h}} 13^{\mathrm{m}} 2 \mathrm{~g}^{\mathrm{s}} 31$

$+7.8 n=14.88,9^{14} 13^{\mathrm{n} 5} 59^{\mathrm{s}} 6 \mathrm{j}$

$+8.2 \pi=14.70,9^{4} 10^{\mathrm{m}} 25^{\mathrm{s}} 45$

$+7.8 \pi=14.01, \quad 81566^{\mathrm{m}} 35.964$ 


\begin{tabular}{|c|c|}
\hline & (Eph. 一 \\
\hline Mars & $\alpha=\underset{22}{24 \mathrm{~h}} 0^{2}{ }^{2} 45^{853} \mathrm{~d} \alpha=+045$ \\
\hline Weisse 1241 & 22.59 .55 .34 \\
\hline Weisse 76 & 23. 6.37 .90 \\
\hline$\psi^{2}$ Aquarii & 23.11 .34 .72 \\
\hline 97 Aquarii & 23.16 .16 .27 \\
\hline
\end{tabular}

21. Oct. 50 Aquarii

d Aquarii

64 Aquarii

70 Aquarii

Mars

Weisse 1241

Weisse 76

$\psi^{2}$ Aquarii

97 Aquarii

23. Oct. $\sigma$ Aquarii

64 Aquarii

70 Aquarii

Mars

Weisse 1241

Weisse 76

$\psi^{2}$ Aquarii

97 Aquarii

27. Oct. 50 Aquarii

- Aquarii

64 Aquarii

70 Aquarii

Mars

Weisse 1241

Weisse 76

$\psi^{2}$ Aquarii

97 Aquarii

Kremsmünster 1877 Dec. 23.
22.17 .55 .80

22.24 .12 .30

22.32 .51 .63

24.42. 5.78

22.51 .2342

22.59 .55 .38

23. 6.38 .00

23.11.34.71

23.16 .16 .33

22.24 .12 .24

$22.32 .51 .7(1)$

$22 \quad 42.5 .84$

22.52 .46 .52

22.59 .55 .39

23. 6.38 .03

23.11 .34 .82

23.16 .16 .40

22.17 .5593

22.24.12.18

22.32 .51 .73

22.42. 5.82

22.56. 1.28

22.59.55.33

23. 6.38 .05

$2311.34 .80)$

23. 16.16 .38
(Eph. - d)

m. Kr. Z.

$\delta=10^{\circ} 34^{\prime} 29^{\prime \prime} 7 \mathrm{~d} \delta=+7^{\prime \prime} 8 \pi=13^{\prime \prime} 84,8^{\prime \prime} 3^{\mathrm{m}} 15^{\prime \prime} 1 \delta^{\prime}$

10.14 .44 .6

10.13 .59 .6

9.50.53.9

15.42 .30 .4

14. 8.52.3

11. 18.8 .4

10.39 .46 .0

11.11 .58 .6

10.14 .45 .1

10.13 .58 .3

9.50 .55 .3

15. 42.30 .3
10.25 .58 .7

10. 1444.2

10. 13.595

9 อ0.53 โ

15.4230 .1

11.18. 8.7

10.39 .45 .5

11.11 .59 .5

$+0.43 \quad 10 \quad 8.20 .5$

10.1445 .15

10.13 .59 .0

9.5055 .0

15. 42.31 .2

14. 8.52 .7

11.18. 7.9

10.39 .46 .3

11.11 .57 .7

9.30 .46 .9

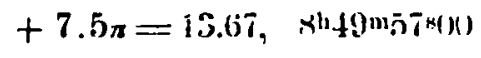

$+7.0 \pi=13.33, \quad 8^{\mathrm{l}} 43^{\mathrm{m}} 28^{\mathrm{s}} 1 \mathrm{~s}$

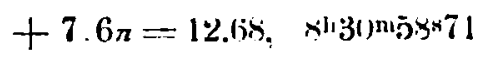

$+0.40$

1i. strasser.

\section{Beobachtungen des Cometen 1873 VII}

auf der provisorischen Universitässternwarte zu Strassburg.

Die Anzeige der Entdeckung dieses Cometen auf | die erste ist, die angestellt wurde; am 10. November der Strassburger Sternwarte am 11. November 1873 findet sich A. N. 1964; es sind dort auch die Beobachtungen vom 11. und 12. Nov. schon mitgetheilt.

Es gelangen spater noch Beobachtungen am 13., 14. und 16. November; die Messungen von letzterem Tage, bei welchen der Comet in kaum $4^{0}$ Höhe beob. achtet werden musste, scheinen die letzten überhaupt vorhandenen zu sein, so wie die Beobachtung vom 11. Nov.

sah man den neuen Cometen in Marseille nämlich „seulement pendant quelques minutes avant son coucher."

Die Strassburger Beobachtungen sind an zwei Ringmikrometern des von mir auch in Karlsruhe gelegentlich zu Cometenbeobachtungen benutzten Fernrohrs von Reinfelder und Hertel von 54"' Oeffnung und 49" Brennweite angestellt. Das Stativ desselben hatte keine Hemmungen und stand auf demselben Bretterfussboden, 